\title{
Selective Chiral Symmetry Breaking during Crystallization: Parity Violation or Cryptochiral Environment in Control?
}

\author{
Cristobal Viedma* \\ Departamento de Cristalografia y Mineralogia, Facultad de Geologia, Universidad Complutense, \\ Madrid, Spain
}

\begin{abstract}
We have attempted to check experimentally whether the parity violating energy difference (PVED) between enantiomers is ultimately responsible for any bias in the final homochirality of crystals, after suitable autocatalytic amplification. When two equivalent (50-50) chiral populations of $\mathrm{L}$ - and D-crystals (of either $\mathrm{NaClO}_{3}$ or $\mathrm{NaBrO}_{3}$ ) generated independently are mixed and undergo dissolution-crystallization, one of the chiral populations disappears randomly in an irreversible autocatalytic competition process that nurtures the other population. If enough experiments are carried out with this 50-50 mixture of chiral crystals, a random distribution of solutions with final L- or D- crystals is obtained in total. However, when the two populations of L- and D-crystals are generated together in the same solution and undergo a dissolution-crystallization process, a nonrandom distribution of the final handedness among different homochiral solutions is obtained. This selective symmetry breaking is sporadic although always in the same direction; thus, we discard any explanation based on PVED effects. We conclude that the result represents an experimental demonstration of selective chiral symmetry breaking on a macrocopic level brought about by cryptochiral environmental impurity.
\end{abstract}

\section{Introduction}

Chiral symmetry breaking occurs when a physical or chemical process, with no preference for the production of one enantiomer or the other, spontaneously generates a large excess of one of two enantiomers: left-handed (L) or right handed (D). The fact that biologically relevant molecules exist only as one of two enantiomers is a fascinating example of complete symmetry breaking in chirality and has long intrigued many scientists. This selective biomolecular chirality or "biochirality" has been connected to a "parity-violating difference" between enantiomers. Until 1957, it was thought that nature was symmetric at the atomic level. However, in that year parity violation (namely, a different probability for the occurrence of a process and its mirror image), as proposed by Lee and Yang, ${ }^{1}$ was discovered in the weak nuclear force: ${ }^{2} \mathrm{~L}$-electrons are preferentially formed relative to D-electrons during the $\beta$-particles decay of radioactive nuclei. The major consequence of this finding is that selective chirality exists at the level of elementary particles. Until the late 1960s, it was assumed that parity violation was confined to nuclear reactions, but then a theory was developed that unified the weak and electromagnetic forces. ${ }^{3}$ An important consequence of this theory is that a chiral molecule exists in a lower or higher energy state relative to its enantiomer. Thus, parity violation results in an energy difference between two species of $\mathrm{L}^{-}$and D-enantiomers. The connection between parity-violating energy difference (PVED) between two enantiomers and the fact of selective biomolecular chirality was first suggested by Yamagata, ${ }^{4}$ who postulated that a small reaction rate or binding energies differences between enantiomers caused by PVED could lead to almost complete selection of one enantiomer during millions of years of evolution. But the magnitude of the PVED between enantiomers is too small to be measured experimentally, and numerous attempts have been made to estimate this magnitude theoretically. The values obtained have ranged from $10^{-5}$ to $10^{-10}$ for crystal lattice energies ${ }^{5,6}$ and $10^{-14}$ for amino acids. ${ }^{7}$ Although the optical rotations of a few atoms

* To whom correspondence should be addressed. E-mail: viedma@geo.ucm.es. have been measured, ${ }^{8,9}$ the experimental confirmation of a PVED between enantiomers is still dubious.

Szabó-Nagy ${ }^{10}$ showed that enantiomeric excess (ee) appeared in the crystallization of the cobalt and iridium complexes. They claimed that this ee was a clear contribution of the PVED between enantiomers in the crystallization process. Nevertheless, this ee was so weak that circular dichoism (CD) signals to measure the optical activities were close to the sensitivity limit of the CD spectrometer.

This means that to reach evident and convincing ee, mechanisms to enhance any initial imbalance in chirality are absolutely essential. ${ }^{11}$ In 1953, Frank ${ }^{12}$ suggested that a form of autocatalysis in which each enantiomer catalyzes its own production, while suppressing that of its mirror image, might have nonlinear dynamics leading to the amplification of small initial fluctuations in the concentrations of the enantiomers. Many theoretical models have been proposed afterward, but they have often been criticized as lacking any experimental support. ${ }^{13}$ Then one of the more versatile and well-studied examples of the symmetry-breaking process was performed by the crystallization of chiral crystals that provides an efficient tool for studying the presumed efficacy of PVED effects experimentally. ${ }^{14-16}$

Thus, chiral symmetry breaking during crystallization is a topic of current interest, and the crystallization of sodium chlorate has played a pivotal role in these studies. ${ }^{17,18}$ The achiral molecules of $\mathrm{NaClO}_{3}$ or $\mathrm{NaBrO}_{3}$ crystallize as two enantiomeric chiral crystals ${ }^{19}$ in the cubic space group $P 2_{1} 3$. Hence, $\mathrm{NaClO}_{3}$ and $\mathrm{NaBrO}_{3}$ are achiral before crystallization, as they exist in solution as more or less dissociated ions or clusters without a fixed chirality, but form chiral crystals. When sodium chlorate or bromate crystallize from water in the absence of any perturbation (unstirred solution) generates a racemic mixture of L- and D-crystals. When the solution is stirred during crystallization, however, a given experiment will yield crystals with the same chirality, either levo or dextro, that is to say complete symmetry breaking or total "crystal enantiomeric excess" (CEE).$^{20}$ If enough crystallizations are carried out under stirring, a random distribution of L- and D- crystals is obtained in total. The usual explanation for this random chiral symmetry 
breaking is secondary nucleation by which a randomly generated single chiral crystal or mother crystal triggers at a fast rate if the solution is stirred the production of a large number of secondary crystals that are enantiomerically identical to itself. The result of this crystallization process is the generation of crystals with the same handedness in a particular solution. Obviously, the handedness of crystals in different solutions is random. ${ }^{20}$

But recently we have described a new symmetry breaking process: We show experimental data indicating that complete symmetry breaking and chiral purity can be achieved from an initial system where both enantiomers are present since the beginning. This is an experimental case in which one observes the complete elimination of a chiral population of crystals of a hand in favor of the other one. ${ }^{21}$ In that work, we show with laboratory experiments how a solution of $\mathrm{NaClO}_{3}$ with a large population of L- and D-crystals moves into complete chiral purity: (i) any small initial crystal ee eventually gives rise to total crystal purity with the less abundant enantiomer disappearing; (ii) "symmetric" proportion of both enantiomeric crystals (a 50-50 mix of chiral crystals) gives rise to total symmetry breaking and crystal purity with one of the two enantiomers disappearing randomly.

We stated ${ }^{21}$ that in our systems this process becomes possible by the combination of (i) a nonlinear autocatalytic dissolutioncrystallization process due to the combined abrasion-grinding (glass balls) and stirring in our experiments; and (ii) the recycling of crystallites when they reach the achiral molecular level in the competitive dissolution-crystallization phenomenon. Thus, complete chiral purity can be achieved. Up to now, there are two other theoretical studies to explain our experiments. ${ }^{22,23}$

However, in these experiments (even a "symmetric" proportion of chiral crystals) the initial population of crystals of every sample was always formed by weighing and mixing $\mathrm{L}-$ and D-crystals obtained separately from different supersaturated solutions. Thus, they are "artificial" or "forced" populations of crystals. We soon noticed that this way to generate racemic populations of chiral crystals had a profound impact on the chiral results of our experiments: "any small difference between Land D-crystals induces the preferred production of one of them; for example small differences in the quality of the crystals bias the progressive enantiomeric amplification of a certain handedness". ${ }^{21}$ Thus, any small chemical-physical fluctuation in the particular history of every solution has a direct effect on crystals generated from it, and this subtle difference between crystals is enough to bias the results of the experiments when crystals of different solutions are mixed. With that experimental protocol, it was impossible to check any other source of possible chiral bias as being a parity-violating difference between enantiomers or any other source of chiral bias or chiral contamination. To obviate this inconvenience, in this research we designed new studies in which experiments of symmetry breaking were performed with initial racemic mixtures of L- and D-crystals obtained spontaneously from the same solution and then under identical circumstances competing between them. The racemic mixture is now the whole and "natural" population of $\mathrm{L}$ - and D-crystals generated together at the same time in the evaporation process of every particular solution.

From the viewpoint of energy, the two chiral crystals of these racemic mixtures can exist with an equal probability, but contradicting this theoretical prediction the chiral result of the symmetry breaking process is now nonrandom.
Table 1. Number of Solutions of $\mathrm{NaClO}_{3}$ with Initial Populations of L- and D-Crystals That Show Complete Symmetry Breaking and Final Chiral Purity (only L- or D-Crystals)

\begin{tabular}{ccccc}
\hline run & $\begin{array}{c}\text { no. of } \\
\text { solutions }\end{array}$ & $\begin{array}{c}\text { solutions with } \\
\text { L-crystals }\end{array}$ & $\begin{array}{c}\text { solutions with } \\
\text { D-crystals }\end{array}$ & $\begin{array}{c}\text { solutions with } \\
\text { L-crystals (\%) }\end{array}$ \\
\hline 1 & 200 & 160 & 40 & 79.5 \\
2 & 240 & 236 & 4 & 98.3 \\
3 & 200 & 102 & 98 & 51 \\
4 & 100 & 83 & 17 & 83 \\
5 & 100 & 49 & 51 & 49 \\
6 & 100 & 73 & 27 & 73
\end{tabular}

\section{Experimental Section}

We prepared several different solutions of $\mathrm{NaClO}_{3}$ by dissolving 5 $\mathrm{g}$ of $\mathrm{NaClO}_{3}$ (from Merck, Sigma-Aldrich, Fluka, Across, Panreac and Riedel-deHaën in different experiments) in $6 \mathrm{~mL}$ of water (bi-distilled water or deionized water, Milli Q-system, in different experiments) and several different solutions of $\mathrm{NaBrO}_{3}$ by dissolving $4 \mathrm{~g}$ of $\mathrm{NaBrO}_{3}$ (from Carlo Erba, Merck, Aldrich, Fluka, Acros, Panreac and RiedeldeHaën in different experiments) in $12 \mathrm{~mL}$ of bi-distilled water and deionized water, Milli Q-system, in different experiments). In some experiments, $\mathrm{NaClO}_{3}$ and $\mathrm{NaBrO}_{3}$ of every source were previously purified by recrystallization two times. The resulting solutions were constantly stirred and heated to $100{ }^{\circ} \mathrm{C}$ to ensure complete dissolution of the solute and then cooled to $60^{\circ} \mathrm{C}$. Then solutions were transferred to Petri dishes of $10 \mathrm{~cm}$ in diameter and $1 \mathrm{~cm}$ high. To obtain a statistically relevant number of L- and D-microcrystals with the same size, these Petri dishes were placed (distance: $60-70 \mathrm{~cm}$ ) under a continuous flow of air generated by a ventilator of $60 \mathrm{~W}$ for at least $24 \mathrm{~h}$. The initial concentration of solutions was such that cooling alone did not produce any crystals, and evaporation of the solution was necessary. Crystallizations were carried out in the dark at room temperature $\left(22-26^{\circ} \mathrm{C}\right)$ and in three different laboratories (although in the same building). After the evaporation process, every Petri dish showed an apparently racemic or "symmetric" mixture of both L- and D-crystals (between 300 and 500 isometric microcrystals for $\mathrm{NaBrO}_{3}$ and 400-700 microcrystals for $\mathrm{NaClO}_{3}$ ). The handedness of the chiral crystals was determined by their optical activity using a petrographic microscope. Following the experimental protocol described ${ }^{18}$ previously, crystals of every Petri dish were gathered and ground to a fine powder using an agate pestle (almost all systems develop efflorescences on the wall of Petri dishes that were not considered in the evaluation). Then this fine powder was placed in $50 \mathrm{~mL}$ round-bottom flasks with $6 \mathrm{~g}$ of small glass balls $(3-5 \mathrm{~mm}$ of diameter). We added $3 \mathrm{~mL}$ of water to flasks with $\mathrm{NaClO}_{3}$ and $5 \mathrm{~mL}$ to flasks with $\mathrm{NaBrO}_{3}$, which only partially dissolved the crystals. At the same time, the whole system (solution, balls, and crystals) was stirred by a magnetic bar at 700$900 \mathrm{rpm}$. After $24 \mathrm{~h}$, total symmetry breaking and complete chiral crystal purity were achieved in the population of the crystals that remained in solution in every system (every solution contained only Lor D-crystals). The experiments were repeated during successive days until a statistically relevant number of data was gathered. Then, trials or runs of this experimental protocol were sporadically repeated during almost 2 years. Quality control at the laboratory was done by randomly checking solutions by polarimetry to detect any chiral impurity.

\section{Results and Discussion}

The handedness of the chiral crystals that remained in solution in the different systems is not random, but almost always there is a predominance (overwhelming sometimes) of L-crystals (in the best cases $99.5 \%$ of solutions with L-crystals). Nevertheless, there are some runs that do not reflect any preference in the handedness of the crystals. That is to say, the selective symmetry breaking seems to be sporadic although always in the same direction (Tables 1 and 2).

Although the assignment of chirality to crystals is arbitrary, we know that both $\mathrm{NaClO}_{3}$ and $\mathrm{NaBrO}_{3}$ show the same selective chiral symmetry breaking (the same handedness of atomic arrangement wins the competition in both products) because the same structural handedness of chiral crystals of both 
Table 2. Number of Solutions of $\mathrm{NaBrO}_{3}$ with Initial Populations of L- and D-Crystals That Show Complete Symmetry Breaking and Final Chiral Purity (only L- or D-Crystals)

\begin{tabular}{ccccc}
\hline run & $\begin{array}{c}\text { no. of } \\
\text { solutions }\end{array}$ & $\begin{array}{c}\text { solutions with } \\
\text { L-crystals }\end{array}$ & $\begin{array}{c}\text { solutions with } \\
\text { D-crystals }\end{array}$ & $\begin{array}{c}\text { solutions with } \\
\text { L-crystals (\%) }\end{array}$ \\
\hline 1 & 260 & 258 & 2 & 99.2 \\
2 & 280 & 274 & 6 & 97.8 \\
3 & 200 & 89 & 111 & 44.5 \\
4 & 100 & 80 & 20 & 80 \\
5 & 100 & 52 & 48 & 52 \\
6 & 100 & 68 & 32 & 68
\end{tabular}

chemicals rotates light in the opposite direction ${ }^{24}$ (like many amino acids). These experiments are examples of complete symmetry breaking with total chiral purity in every solution and, at the same time, selective chirality (sporadically) between the different solutions that show crystals with the same structural handedness. During a few months (runs 1-2 of every chemical), we maintained the hypothesis that this selective symmetry breaking could be consequence of PVED between enantiomers.

But the last runs of the experiments have disputed this hypothesis. Since the PVED will always operate in the same direction, the selective symmetry breaking will always be in the same direction. If instead a crypto impurity is the defining factor, it should be common to all the samples and thus result in symmetry breaking in the same direction, but not necessarily in the same direction of the PVED effect. However, if no crypto impurities are present and PVED is ineffective, solutions have a few more of one enantiomer than the other randomly, as any racemic mixture, and will have random chiral symmetry breaking. The fact that the selective symmetry breaking is not continuous but intermittent suggests that either the chiral bias cannot be based on intrinsic deterministic forces as PVED or its possible effects are masked. This last consideration could be plausible if PVED and the crypto impurity operate in the opposite direction. Thus, the most plausible explanation to the nonstochastic distribution of handedness in these symmetry breaking experiments is the sporadic presence of chiral crypto impurities.

Singleton and Loan, ${ }^{25}$ by an adaptation of the asymmetric autocatalysis reactions of Soai, showed that undetected trace amounts of optically active materials may dominate the outcome of reactions involving asymmetric autocatalysis. Although efforts to detect the optically active impurities in these reactions were not successful, their source could be probed experimentally. They proved that there were not heterogeneous contaminants arising from dust or the reaction flask surfaces or introduced from syringes. The major source of optical activity was homogeneous in the organic starting materials. Simply changing starting material makes a difference between pairs of trials that generate one or the other enantiomer.

Our systems are much simpler, and there are not organic material or chiral molecules involved in experiments. We have attempted to avoid any chiral impurity by changing the experimental conditions and basically altering the source of the starting materials. But despite this effort the phenomenon of selective chiral symmetry breaking persists in many of our experiments. However, it is an adage in chemistry that "purity is a matter of degree." Solutions or environments that contain optically active impurities, present in concentrations well below the level of detection, may be referred to as cryptochiral. ${ }^{26}$

It seems that we are dealing with a cryptochiral environment that induces selective chiral symmetry breaking. It is well-known that nuclei or seeds of a given phase may contaminate a laboratory (or even a building) and predetermine all following crystallizations to yield even the same chiral phase. The effect of impurities on nucleation and crystal growth is of fundamental importance to the kinetics of crystallization: ${ }^{27}$ If the impurity is chiral, the enantiomers of the crystallizing compound may nucleate and crystallize at different rates.

Thus, a likely explanation for the nonstochastic distribution of handedness in these crystallizations is the presence of optically active impurities that may either favor the formation of L-nuclei or disfavor the growth of D-crystals by selective surface adsorption. ${ }^{28}$

Crystal nucleation may be achieved at the air-aqueous solution interface, acting as a templating agent, and chiral impurities, present in the surface, can bias the process. On the other hand, using the Langmuir adsorption theory, Kubota and Mullin have formulated a theory to describe the retardation of the growth rate by the adsorbed impurity. ${ }^{29}$ This theory is based on the idea that the adsorbed impurity interferes with the orderly growth process of the crystal surface. Chiral impurities could be adsorbed on the different enantiomers surface at different rates.

Nevertheless, in all traditional experiments performed in the last 10 years, ${ }^{20,21,30-33}$ no bias has been found in the crystallization of $\mathrm{NaClO}_{3}$ or $\mathrm{NaBrO}_{3}$. Stirred crystallization from a supersaturated solution generates either L- or D-crystals, but the dominance of one or another enantiomer in a particular crystallization is entirely random. When a solution is not stirred, statistically equal numbers of L- or D-crystals are generated.

During the last several years, all these experiments have been reproduced in our laboratory without detecting any bias in Land D-crystal production. ${ }^{21,33}$ These experiments have been repeated again from the same sources (even from the same flasks) of the products used in this work and at the same time with similar results of no preference in chiral crystals production. Nevertheless, when classical stirring experiments are performed under a strong flow of air, the systems produce slightly more L- than D-crystals but very near statistical error (unpublished data).

Traditional symmetry breaking in crystallization is produced by the appearance of one single L- or D- "mother crystal" that seeds the solution. This means that any effect of a cryptochiral environment on this crystallization should be by the influence on this single event of nucleation by the mere contact of static air on the surface of the solution. Nevertheless, the experimental protocol used in our systems has the following differences and advantages:

First, during all these new experiments there is a strong flow of air over the surface of the solution that enhances the incorporation of hypothetical foreign chiral molecules into the systems.

Second, in every solution there is not only one nucleation event but several hundred nucleations that is much more relevant from a statistical point of view to check any small difference in the generation of both enantiomers induced by a cryptochiral environment. The whole population of these crystals undergoes the symmetry breaking process.

Third, during the growth process L- and D-crystals compete for the solute in every solution. The growth rate between both enantiomers could be slightly altered by impurity selective adsorption on the crystal surface. It is necessary to remark here that the most important parameter in our symmetry breaking process is not the initial number of crystals (they are ground to a fine powder) but the "total mass" or weigh of every chiral phase as a whole. Thus, the growth rate could be a fundamental step.

Singleton and Loan $^{34}$ showed experimentally that a very low ee $\left(10^{-16} \mathrm{M}\right)$ was sufficient to control the enantiomeric outcome 
in their reactions. The essence of our nonlinear autocatalyticrecycling process is the competition between millions of microcrystals in a continuous dissolution-crystallization phenomenon: we are dealing with the perfect scenario in which any small difference between enantiomers due to any chiral force or chiral impurity can be checked. Then, the combination of these three factors described could generate a slight ee in solution which by amplification of the nonlinear autocatalytic dissolution-crystallization process induces the selective symmetry breaking here reported. Perhaps because of these differences, no bias has been detected in stirred solutions that generate only one single initial L- or D-mother crystal.

Whichever the origin of the cryptochiral environment, either of biological origin or nuclei seeds of a given chirality that contaminates the building or even a cryptochiral environment as the result of three billion years of life, it is clear that the symmetry breaking process described here is much more sensitive than any other process of symmetry breaking in crystallization reported in the literature.

We are dealing with a powerful experimental protocol that detects chiral bias between enantiomers, which never before has been detected, and this small chiral bias is amplified to absolute chiral purity. That could be in future research an efficient tool to check any chiral bias in many systems.

Finally, it is tempting to speculate on positive implications of our work on the question of the origin of biochirality. We have shown that both the crystallization of enantiomers and the autocatalytic symmetric breaking process in crystallization with amplification of chirality are highly enantioselective as well as sensitive to a cryptochiral environment. This indicates ${ }^{35}$ how homochirality may propagate from one compound to another and from one location to another, which is a plausible mechanism in a theory describing the origin of biomolecular homochirality.

\section{References}

(1) Lee, T. D.; Yang, C. N. Phys. Rev. 1956, 102, 290

(2) Wu, C. S.; Ambler, E.; Hayward, R. W.; Hoppes, D. D.; Hudson, R. P. Phys. Rev. 1957, 105, 1413.
(3) Weinberg, S. Phys. Rev. Lett. 1967, 19, 1264.

(4) Yamagata, Y. J. J. Theor. Biol. 1966, 11, 495.

(5) Thiemann, W.; Wagener, K. Angew. Chem., Int. Ed. Engl. 1970, 9, 740.

(6) Rein, D. W. J. Mol. Evol. 1974, 4, 15.

(7) Mason, S. F.; Tranter, G. E. Chem. Phys. Lett. 1983, 94, 34.

(8) Macpherson, M. J. D.; Zetie, K. P.; Warrington, R. B.; Stacey, D. N.; Hoare, J. P. Phys. Rev. Lett. 1991, 67, 2784.

(9) Warrington, R. B.; Thompson, C. D.; Staney, D. N. Europhys. Lett. 1993, 24, 641.

(10) Szabo-Nagy, A.; Keszthelyi, L. Proc. Natl. Sci. U.S.A. 1999, 96, 8, 4252.

(11) Schmidt, J. G. P.; Nielsen, E.; Orgel, L. E. J. Am. Chem. Soc. 1997, 119, 494.

(12) Frank, F. C. Biochem. Biophys. Acta 1953, 11, 459.

(13) Bonner, W. A. Top. Stereochem. 1988, 18, 1.

(14) Bonner, W. A. Orig. Life Evol. Biosph. 1994, 25, 401.

(15) Bonner, W. A. Orig. Life Evol. Biosph. 1996, 26, 27.

(16) Bonner, W. A. Orig. Life Evol. Biosph. 1988, 29, 317.

(17) Pagni, R. M.; Compton, R. N. Cryst. Growth Des. 2002, 2, 249.

(18) Niedermaier, T.; Schlenk, W., Jr. Chem. Ber. 1972, 105, 3470.

(19) Abrahams, S. C.; Bernstein, J. L. Acta Crystallogr. 1977, B33, 3601.

(20) Kondepudi, D. K.; Kaufman, R. J.; Singh, N. Science 1990, 250, 975.

(21) Viedma, C. Phys. Rev. Lett. 2005, 94, 065504.

(22) Uwaha, M. J. Phys. Jpn. 2004, 73, 10, 2601.

(23) Saito, Y.; Hyuga, H. J. Phys. Soc. Jpn. 2005, 74, 535.

(24) Abrahams, S. C.; Glass, A. M.; Nassau, K. Solid State Commun. 1977, 24, 515.

(25) Singleton, D. A.; Vo, L. K. J. Am. Chem. Soc. 2002, 124, 10010.

(26) Mislow, K.;. Czech. Collect. Chem. Commun. 2003, 68, 849.

(27) Kondepudi, D. K.; Crook, E. K. Cryst. Growth Des. 2005, 5, 2173.

(28) Weissbuch, I.; Lahav, M.; Leiserowitz, L. Cryst. Growth Des. 2003, 3,125 .

(29) Kubota, N.; Mullin, J. W. J. Cryst. Growth 1995, 152, 203-208.

(30) Kondepudi, D. K.; Bullock, K. L.; Digits, J. A.; Hall, J. K.; Miller, J. M. J. Am. Chem. Soc. 1993, 115, 10211.

(31) Kondepudi, D. K.; Bullock, K. L.; Digits, J. A.; Yarborough, P. D. J. Am. Chem. Soc. 1995, 117, 401.

(32) Martin, B.; Tharrington, A.; Wu, X-1. Phys. Rev. Lett. 1996, 77, 2826.

(33) Viedma, C. J. Cryst. Growth 2004, 261, 118.

(34) Singleton, D. A.; Vo, L. K. Org. Lett. 2003, 5, 4337.

(35) Vestergren, M.; Johansson, A.; Lennartson, A.; Hakansson, M. Mendeleev Commun. 2004, 1, 258. 